\title{
3 Wahn und Kultur
}

\author{
Thomas Stompe und Hans Schanda
}

\begin{abstract}
Kulturvergleichende Untersuchungen können fundamentale Erkenntnissen über das Wesen und die Bedingungsfaktoren psychischer Erkrankungen, über natürliche und kulturell überformte Verlaufsformen und damit auch über Behandlungsoptionen liefern. Dieser Forschungszweig der Psychiatrie ist allerdings mit erheblichen Problemen konfrontier. Dazu gehören u.a. die Auswahl bzw. Neukonzeptionalisierung von psychopathologischen Begriffen, die Auswahl bzw. Schaffung von geeigneten Erhebungsinstrumenten, die Schulung der beteiligten Forscher und der Erwerb von interkultureller Kompetenz. Der potenzielle Ertrag der kulturvergleichenden Methode soll am Beispiel des Wahns dargestellt werden.
\end{abstract}

\subsection{Wahndefinitionen und kulturvergleichende Untersuchungen}

Die Denkinhalte in verschiedenen Kulturen und Ethnien unterscheiden sich mitunter erheblich. Wie sehr an Auffassungen festgehalten wird, unterscheidet sich ebenfalls deutlich von Kultur zu Kultur. Die formalen Denkabläufe hingegen sind erstaunlich ähnlich: in allen Kulturen werden Kategorien und semantische Netzwerke gebildet; die syntaktische Tiefenstruktur gehorcht ähnlichen Gesetzen, die logischen Gesetze (z.B.: Aussage-, Prädikanten- oder Diskurslogik) können auf alle Sprachen angewandt werden. Die sprachlichen Kontexte und die Sprechakte unterscheiden sich allerdings von Kultur zu Kultur. Daraus ergibt sich, dass nur die Wahndefinitionen für kulturvergleichende Untersuchungen geeignet sind, welche den Wahn über Störungen der formalen 
Denkabläufe definieren (Stompe 2010). Am wenigsten geeignet sind hingegen Definitionen, die sich auf das 3. Jaspers'sche Wahnkriterium, die „Unmöglichkeit des Inhalts“, beziehen. Daneben sollte durch eine Wahndefinition ein möglichst breites Spektrum holothymer und parathymer Wahninhalte erfasst werden. Bis in die 1980er-Jahre waren Wahndefinitionen vorwiegend kategorial. In den letzten 20 Jahren mehrten sich die Bemühungen, einen dimensionalen Zugang zum Wahn zu finden. Basis dafür ist das Postulat eines Kontinuums von „normalem“ zu „wahnhaftem“ Denken. Außer acht gelassen wurde dabei oft die Tatsache, dass ein dimensionaler Zugang definitorische Probleme nicht beseitigt, sondern nur den zu definierenden Bereich verlagert: während ein kategorialer Ansatz den Bruch zwischen „normalem“ Denken und Wahn definitorisch erfassen muss, steht ein dimensionaler Zugang vor der Aufgabe, entweder den Skalenanfang und das Skalenende, oder einen Cut-off Punkt zu definieren bzw. die semantische Bedeutung eines Schnittpunktes von zwei oder mehreren Skalen inhaltlich-hermeneutisch zu erfassen.

\subsubsection{Für kulturvergleichende Untersuchungen ungeeignete Wahndefinitionen}

Nach Jaspers (1913) sind die äußeren Merkmale der Wahnideen:

1. Die außergewöhnliche Überzeugung, mit der an ihnen festgehalten wird, also die unvergleichliche subjektive Gewissheit.

2. Die Unbeeinflussbarkeit durch Erfahrung und zwingende Schlüsse.

3. Die Unmöglichkeit des Inhalts.

Durch das dritte Kriterium ist es unmöglich, zwischen kulturtypischen Krankheitskonzepten, für uns ungewöhnlichen religiösen Vorstellungen und Wahnthemen zu unterscheiden. Viele Forscher nach Jaspers beriefen sich ebenfalls auf den Aspekt der Unmöglichkeit des Inhalts. Nach Huber und Gross (1977) beruht Wahn auf inhaltlich falschen, nicht aus anderen Erlebnissen ableitbaren Überzeugungen, die mit apriorischer Evidenz auftreten und an denen bei erhaltener Intelligenz trotz der Unvereinbarkeit mit dem bisherigen Erfahrungszusammenhang und der objektiv nachprüfbaren Realität festgehalten wird. Die American Psychiatric Association definierte Wahn im Handbuch des DSM-IV ähnlich:

„Wahnphänomene sind falsche Überzeugungen, die gewöhnlich mit einer Fehldeutung von Wahrnehmungen und Erfahrungen einhergehen ... Ideen, die in einem Kulturkreis als wahnhaft erscheinen, können in einem anderen üblich sein“"(Saßet al. 1996).

\subsubsection{Mäßig geeignete Wahndefinitionen}

Nach Eugen Bleuler (1911), der den Begriff Schizophrenie in die psychiatrische Nomenklatur eingeführt hat, entstehen Wahnideen durch Affektwirkung, haben immer eine bestimmte affektgetragene Richtung und sind der Korrektur 
durch neue Erfahrungen oder Belehrungen unzugänglich. Im Gegensatz dazu Peter Berner (1982): „Wahn ist der Ausschluss des Zufalls bei fehlender Affekteinengung“ oder der Philosoph Manfred Spitzer (1989):

„Beim Wahn handelt es sich um Aussagen, die mit der Sicherheit von Aussagen über eigene mentale Zustände geäußert werden, bei deren Inhalt es sich aber nicht um mentale Zustände, sondern um intersubjektiv zugängliche Sachverhalte handelt.“

\subsubsection{Gut geeignete Wahndefinitionen}

Eine für kulturvergleichende Untersuchungen gut geeignete Wahndefinition stammt von Kraepelin (1899), einem der Begründer der modernen Psychiatrie: „Wahnideen sind Störungen des Urteils und der Schlussbildung“. Zwanzig Jahre später Jossmann (1921): „Im Wahn tritt der Anspruch auf objektive Geltung einer an sich subjektiven Wertung und das Nicht-von-mir-Ausgehen dieser Geltung im Erleben auf“. Nach Gruhle (1951) ist der Wahn Beziehungssetzung ohne Anlass; Blankenburg (1991) meint, dass pathologisches Bedeutungserleben durch die Unfähigkeit gekennzeichnet ist, empfangene Eindrücke integrativ zu verarbeiten und mitzuteilen; nach Janzarik (1988) ist Wahn eine die Zufälligkeit oder nur subjektive Gültigkeit ausschließende Überzeugung, solange sie sich einer sie aufhebenden Desaktualisierung entzieht; und nach Conrad (1958) ist Wahn durch einen Verlust an Freiheitsgraden bestimmt. Der fundamentale Wechsel des Bezugssystems, der Überstieg, kann nicht mehr vollzogen werden. Dies führt zum Gefühl, im Mittelpunkt zu stehen und zu abnormen Bedeutungsideen. Unsere Arbeitsgruppe entwickelte für Studien zu transkulturellen Aspekten von Wahnthemen eine formale Wahndefinition, die bewusst alles Inhaltliche wie Bizarrheit oder Unmöglichkeit des Inhalts ausklammert und ihre Wurzeln in der transzendentalen Philosophie Kants sowie der analytischen Philosophie (und hier speziell in der nordamerikanischen Richtung der Philosophy of Mind) hat: Eine Wahnidee ist ein synthetisches Apriori als Konklusion eines induktiven Schlusses. Unter synthetisch versteht man im Gefolge von Kant Bezeichnungen für Sätze, die nicht allein aufgrund logischer und definitorischer Vereinbarungen gelten, also nicht analytisch sind, sondern nur in Bezug auf nicht-sprachliche Sachverhalte begründet werden können. Zu unterscheiden sind nach Mittelstraß (1995) empirische (a posteriori) von nicht-empirisch begründbaren und dennoch sachhaltig-erkenntniserweiternde synthetische Urteile a priori.

Dem amerikanischen Philosophen Robert Brandom (2004) folgend erweist sich der Realitätsgehalt eines theoretischen Objekts dann, wenn es möglich ist, dieses mit mindestens zwei unterschiedlichen theoretischen Ansätzen reliabel zu erfassen. Für (kulturvergleichende) Untersuchungen zum Wahn bedeutet das, dass das Vorliegen eines Wahns dann als gesichert gelten kann, wenn unter Verwendung von zwei Wahndefinitionen mit unterschiedlichem theoretischen Hintergrund (z.B.: Wahn nach Conrad, bzw. Spitzer etc.) die Diagnose eines Wahns gestellt werden kann. 


\subsection{Wahninhalte}

Die Schwierigkeiten der Konzeption kulturvergleichender Wahnstudien enden allerdings nicht damit, sich auf eine Wahndefinition zu einigen, auch zur Frage, welche Inhalte ein Wahn haben kann, gibt es sehr unterschiedliche Auffassungen. Diese Problematik wurde bis in das frühe zwanzigste Jahrhundert hinein heftig diskutiert. Prominente Nervenärzte wie Esquirol, Capgras oder Fregoli vertraten die Auffassung, dass jeder Wahninhalt eine nosologisch eigenständige Erkrankung sei. Im 20. Jahrhundert setzten sich nur wenige Psychiater systematisch mit der Erforschung der Prävalenz von Wahnthemen auseinander. Die Zahl der in der kulturvergleichenden Psychiatrie erhobenen Wahnthemen schwankt je nach Autor zwischen 6 und 21 (Kala u. Wig 1982; Kim et al. 2001; Masłowski 1986; Ndetei u. Vadher 1984; Suhail u. Cochrane 2002; Tateyama et al. 1993, 1998). Blankenburg (1991) wies darauf hin, dass es sich bei den gängigen Einteilungen um eine Aufzählung von völlig Heterogenem handelt. Ganz verschiedene Einteilungsprinzipien sind am Werk, die Kategorien überschneiden sich in verschiedener Weise, Wesentliches und Unwesentliches sind durcheinander gewürfelt. Solange es nicht gelingt, vernünftige Einteilungsprinzipien zu finden und eine Ordnung der Wahninhalte herzustellen, wird die vergleichende Psychopathologie in diesem Bereich kaum Fortschritte machen.

Im Folgenden soll ein semantisch-phänomenologisches Modell vorgestellt werden, mit dessen Hilfe Ordnung in die Vielzahl der möglichen Wahninhalte gebracht werden kann (s. Abb. 10).

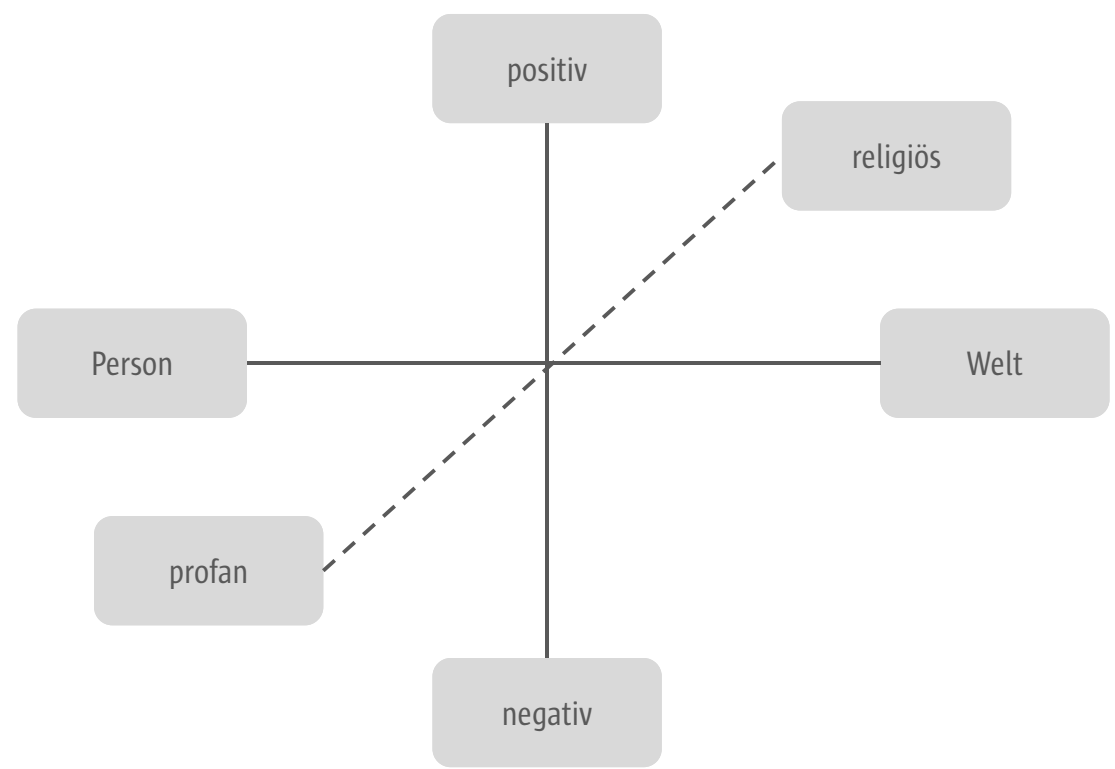

Abb. 10 Semantisch-phänomenologische Dimensionen der Wahnthemen 
Im Wahn können entweder die Welt, die Person oder beide Bezugssysteme als verändert erlebt werden. Unter „Person“ wird die Gesamtheit der körperlichen, sozialen, psychischen und Identitätsbezüge eines Menschen, unter „Welt“ die soziale Mitwelt, aber auch die geschichtliche Situation, die physische und transzendente Außenwelt verstanden. Die wahnhafte Veränderung des Bezugsystems Welt-Person ist in ihrer affektiven Qualität nie neutral, sondern immer positiv oder negativ gefärbt. „Positiv-negativ“ umfasst dabei zahlreiche Dichotomien wie „gut-böse“, „schön-hässlich“, „arm-reich“, „,bedrohlich-beschützend“, „groß-klein“, „gesund-krank“, „verdammt-auserwählt“, etc. Negative, auf eine Veränderung des Welterlebens verweisende Wahninhalte sind Verfolgungs-, Vergiftungs-, Eifersuchts- oder Weltuntergangsideen. Die wichtigste positive Veränderung der Welt im Wahn ist der Liebeswahn, d.h. der Wahn, geliebt zu werden. Negative, auf die eigene Person bezogene Wahninhalte sind Schuldideen, hypochondrische Wahnideen und Verarmungswahn. Positive, personenbezogene Inhalte sind alle Varianten von Größenund Berufungsideen. Wahnthemen können darüber hinaus entweder religiös oder profan sein. Innerhalb dieser inhaltlichen Dimensionen des Wahns konkretisieren sich in der Psychose alle anthropologisch bedeutungsvollen Daseinsthemen wie Bedroht- oder Auserwähltsein, Liebe und Verlust, die Unversehrtheit des Leibes, Schuld und Scham, Weltanfang und Weltende, oder das Sein im Angesicht des Göttlichen.

\subsubsection{Die Prävalenz von Wahnthemen im Kulturvergleich}

Nach diesen theoretischen Vorüberlegungen wurde 1995 die „International Study on Psychotic Symptoms“ konzipiert, an der sich bisher insgesamt 10 Länder beteiligen (Stompe et al. 1999a, 2006a, 2006b, 2007; Stompe u. Friedmann 2007). Patienten mit Schizophrenie nach DSM-IV (SCID 1) wurden mit einem semi-strukturierten Fragebogen über Wahninhalte, Halluzinationen und Schneider'sche Erstrangsymptome befragt (Phänomenologie, Punktprävalenz; 12-Monatsprävalenz; Lebenszeitprävalenz). Ausgewertet und veröffentlicht wurden bisher die Daten von 1.o8o Patienten aus 7 Ländern (Österreich; Polen, Litauen, Georgien, Nigeria, Ghana, Pakistan). Der Vergleich der Lebenszeitprävalenz und der Punktprävalenz der Wahnthemen der einzelnen Länder zeigt auf den ersten Blick mehr Unterschiede als Gemeinsamkeiten (s. Tab. 5).

Mit einer Lebenszeitprävalenz zwischen 66,2\% (Georgien) und 98,7\% (Ghana) war Verfolgung in allen Ländern das häufigste Wahnthema. Die Punktprävalenz, das heißt die Aktualität eines Themas zum Zeitpunkt der Befragung, liegt noch immer zwischen $57,4 \%$ (Österreich) und 85,4\% (Pakistan). Das bedeutet, dass Bedrohungsszenarien im Verlauf der Erkrankung sehr häufig erlebt werden, bzw. eine sehr starke Neigung zur Chronifizierung zeigen. Die Verfolgungsthematik scheint das Produkt der kognitiven Verarbeitung der emotionalen Erfahrung am Beginn der Psychose zu sein. In der Wahnstimmung gewinnt die umgebende Welt zumeist einen unbestimmt bedrohlichen 
Tab. 5 Lebenszeit- und Punktprävalenz von schizophrenen Wahnthemen in \% ( $N=1.080)$

\begin{tabular}{|c|c|c|c|c|c|c|c|}
\hline & $\begin{array}{l}\text { Österreich } \\
(\mathrm{N}=350)\end{array}$ & $\begin{array}{l}\text { Polen } \\
(\mathrm{N}=80)\end{array}$ & $\begin{array}{l}\text { Litauen } \\
(\mathrm{N}=73)\end{array}$ & $\begin{array}{c}\text { Georgien } \\
(\mathrm{N}=74)\end{array}$ & $\begin{array}{l}\text { Pakistan } \\
(\mathrm{N}=103)\end{array}$ & $\begin{array}{c}\text { Nigeria } \\
(\mathrm{N}=324)\end{array}$ & $\begin{array}{c}\text { Ghana } \\
(\mathbb{N}=76)\end{array}$ \\
\hline \multicolumn{8}{|c|}{ Lebenszeitprävalenz } \\
\hline Verfolgung & $82,0 \%$ & $90,0 \%$ & $98,6 \%$ & $66,2 \%$ & $91,3 \%$ & $77,1 \%$ & $98,7 \%$ \\
\hline Größe & $43,4 \%$ & $41,3 \%$ & $42,5 \%$ & $37,8 \%$ & $10,7 \%$ & $41,7 \%$ & $64,5 \%$ \\
\hline Religion & $34,6 \%$ & $33,8 \%$ & $30,1 \%$ & $32,4 \%$ & $7,8 \%$ & $37,7 \%$ & $51,3 \%$ \\
\hline Hypochondrie & $17,4 \%$ & $28,8 \%$ & $23,3 \%$ & $4,1 \%$ & $4,9 \%$ & $14,2 \%$ & $27,6 \%$ \\
\hline Schuld & $13,7 \%$ & $18,8 \%$ & $24,7 \%$ & $5,4 \%$ & $1,0 \%$ & $10,5 \%$ & $9,2 \%$ \\
\hline Vergiftung & $10,9 \%$ & $31,3 \%$ & $32,9 \%$ & $32,4 \%$ & $16,5 \%$ & $29,3 \%$ & $39,5 \%$ \\
\hline Apokalypse & $8,3 \%$ & $12,5 \%$ & $32,9 \%$ & $16,2 \%$ & $1,0 \%$ & $18,8 \%$ & $9,2 \%$ \\
\hline Abstammung & $4,6 \%$ & $12,5 \%$ & $15,1 \%$ & $25,7 \%$ & - & $8,3 \%$ & $10,5 \%$ \\
\hline Liebe & $5,4 \%$ & $11,3 \%$ & $4,1 \%$ & $12,2 \%$ & - & $6,5 \%$ & $9,2 \%$ \\
\hline Eifersucht & $2,3 \%$ & $1,3 \%$ & $5,2 \%$ & $6,8 \%$ & $1,9 \%$ & $6,5 \%$ & $2,6 \%$ \\
\hline \multicolumn{8}{|l|}{ Punktprävalenz } \\
\hline Verfolgung & $57,4 \%$ & $73,4 \%$ & $87,7 \%$ & $58,1 \%$ & $85,4 \%$ & $71,9 \%$ & $89,5 \%$ \\
\hline Größe & $13,1 \%$ & $10,0 \%$ & $13,7 \%$ & $14,9 \%$ & $4,9 \%$ & $34,6 \%$ & $52,6 \%$ \\
\hline Religion & $25,7 \%$ & $31,3 \%$ & $23,3 \%$ & $24,3 \%$ & $3,9 \%$ & $33,3 \%$ & $40,8 \%$ \\
\hline Hypochondrie & $6,0 \%$ & $12,5 \%$ & $8,2 \%$ & - & $1,0 \%$ & $10,5 \%$ & $15,8 \%$ \\
\hline Schuld & $3,7 \%$ & $6,3 \%$ & $11,0 \%$ & - & - & $13,7 \%$ & $1,3 \%$ \\
\hline Vergiftung & $4,3 \%$ & $13,8 \%$ & $12,3 \%$ & $18,9 \%$ & $13,6 \%$ & $25,3 \%$ & $28,9 \%$ \\
\hline Apokalypse & $2,3 \%$ & $3,8 \%$ & $15,1 \%$ & $2,7 \%$ & - & $9,6 \%$ & $6,6 \%$ \\
\hline Abstammung & $2,6 \%$ & $1,3 \%$ & $5,5 \%$ & $14,9 \%$ & - & $5,6 \%$ & $6,6 \%$ \\
\hline Liebe & $0,3 \%$ & - & - & $2,7 \%$ & - & $14,3 \%$ & $3,9 \%$ \\
\hline Eifersucht & - & - & $1,4 \%$ & $1,4 \%$ & $1,0 \%$ & $2,8 \%$ & - \\
\hline
\end{tabular}

$\Sigma \%>100 \%$, da Mehrfachnennungen möglich waren

Charakter. Wenn durch die Wahnarbeit das Gefühl der diffusen Bedrohung konkrete Form annimmt, tritt für gewöhnlich eine affektive Stabilisierung und Beruhigung ein.

Ähnlich verhält es sich mit dem Erleben der eigenen Person. Im Kern der schizophrenen Psychose findet sich immer eine implizite oder explizite Größenidee. Wie Klaus Conrad (1958) am Begriff der Apophänie expliziert, erlebt sich der Kranke als Zentrum der Welt. Alles ist speziell für ihn „aufgestellt“. Ob sich aus dieser impliziten Größenidee ein expliziter Größenwahn, ein Schuldwahn oder ein hypochondrischer Wahn entwickelt, hängt von verschiedenen Faktoren wie dem Schwerpunkt des Affektes in der Wahnstimmung aber auch von dem kulturellen Umfeld des Kranken ab. Beim Größenwahn fällt die Diskrepanz zwischen Lebenszeit- und Punktprävalenz auf: wäh- 
rend mit der Ausnahme von Pakistan - nach Verfolgungswahn - Größeninhalte im Gesamtverlauf am zweithäufigsten zu finden sind (10,7-64,5\%), sinkt dieser Wert zum Zeitpunkt des Interviews auf Werte zwischen 4,9\% (Pakistan) und 52,6\% (Ghana). Auffällig sind die großen Unterschiede zwischen Lebenszeit- und Punktprävalenz besonders in den europäischen Ländern (Österreich: $43,4 \%$ vs. $13,1 \%$, Polen: $41,3 \%$ vs. $10,0 \%$, Litauen: $42,5 \%$ vs. $13,7 \%$, Georgien: 37,8\% vs. 14,9\%). Das bedeutet, dass wahnhafte Größenideen in diesen Ländern von vielen Patienten zeitlich begrenzt durchlebt werden und später von anderen auf die Person bezogenen Themen wie Schuldwahn oder hypochondrischem Wahn abgelöst werden. Die Differenz von Lebenszeit- und Punktprävalenz des Größenwahns ist in den außereuropäischen Ländern deutlich geringer: vor allem in den beiden westafrikanischen Staaten zeigen die Patienten auch zum Zeitpunkt des Befragung häufig eine wahnhafte Überhöhung des eigenen Person (Nigeria: 41,7\% vs. 34,6\%, Chana: 64,5\% vs. 52,6\%). In Pakistan dagegen ist Größenwahn generell ein sehr seltenes Ereignis (10,7\% vs . 4,9\%).

Ungewöhnlich häufig finden sich religiöse Wahninhalte bei der Schizophrenie (Huber u. Gross 1977; Tateyama et al. 1993). Während die religiöse Komponente im depressiven Schuldwahn in den letzten 100 Jahren nahezu vollständig verschwunden ist (Kranz 1955; Lenz 1967), hat sich die (europäische) Säkularisierung nur wenig auf die Prävalenz des religiösen Wahns in der Schizophrenie ausgewirkt (Stompe et al. 2003). Die phänomenologische Qualität der Wahnstimmung, die in Vielem dem Erleben des Numinosen (Rudolf Otto) ähnlich ist, legt eine Verortung des psychotischen Erlebniswandels in religiöse Symbolsysteme nahe. Eine differenzierte Datenanalyse des religiösen Wahns zeigt allerdings, dass der Einfluss des religiösen Umfelds nicht zu unterschätzt werden darf. Die Lebenszeitprävalenz des religiösen Wahns ist bei Anhängern von Stammesreligionen mit 100\% am höchsten, gefolgt von den westafrikanisch-christlichen $(34,6 \%)$ und den westafrikanisch-islamischen Patienten $(32,5 \%)$. Etwas niedriger war die Prävalenz mit jeweils 30\% bereits bei den europäischen Katholiken und den Georgisch-Orthodoxen, am niedrigsten bei den europäischen Protestanten (20\%), den Atheisten (4\%) und den orthodoxen Sunniten in Pakistan $(7,8 \%)$. Ein ekstatisch-synkretistisches religiöses Umfeld dürfte einen guten Nährboden für religiöse Wahnideen bieten. Dagegen scheint die Bilderfeindlichkeit des dogmatischen Islam und des europäischen Protestantismus die Pathogenese des religiösen Wahns zu hemmen. Obwohl religiöser Wahn vorwiegend im Frühverlauf schizophrener Erkrankungen zu finden ist (Stompe 2008), ist die Diskrepanz zwischen Lebenszeit- und Punktprävalenz weniger deutlich als beim Größenwahn. Vermutlich wird wahnhafte Größe in den meisten Kulturen durch die Konfrontation mit der Realität der Lebenswelt leichter korrigiert als religiöse Ideen, die durchaus auch ängstigenden Charakter wie etwa die Bedrohung durch Dämonen oder die bevorstehende Apokalypse haben können.

Es zeigt sich allerdings deutlich, dass der religiöse Wahn eigentlich kein eigenständiges Wahnthema ist, sondern, ebenso wie Profanität, lediglich eine bestimmte Färbung unterschiedlicher Wahnthemen darstellt. Hypochondrische 
Wahnideen kommen bei etwa 14-28\% der Patienten zumeist nach längerem Krankheitsverlauf vor (Stompe 2008). Ausnahmen sind Georgien und Pakistan mit deutlich niedrigeren Prävalenzen. Schuldwahn findet sich am häufigsten in katholischen Ländern und am seltensten bei den orthodox-sunnitischen Pakistanis (Stompe et al. 2006a). Beide Wahnthemen haben keinen überdauernden Status, die Lebenszeitprävalenz liegt in allen Ländern deutlich über der Punktprävalenz. In Relation zu anderen Wahnthemen hat der Vergiftungswahn eine starke Tendenz zu persistieren.

Die Prävalenz des Weltuntergangswahns hängt zum Teil auch vom kulturellen Hintergrund ab: in anomischen, im Umbau begriffenen Gesellschaften wie etwa Litauen oder Polen finden sich die höchsten Raten, andererseits dürfte der Akuität der Psychose ein zentraler Stellenwert zukommen; in Nigeria, dem Land mit der höchsten Rate an schizoaffektiven Erkrankungen, fand sich auch eine hohe Prävalenz apokalyptischer Ideen (Stompe u. Friedmann 2007). Dass apokalyptische Wahnideen Anzeichen eines akut-psychotischen Geschehens sind und nur in Phasen hoher Wahndynamik vorkommen, zeigt sich in der Relation von Punkt- zu Lebenszeitprävalenz.

Abstammungs-, Liebes- und Eifersuchtswahn gehören zu den seltenen Wahnthemen. Abstammungswahn, eine Variante des Größenwahns, kommt am häufigsten in den osteuropäischen Staaten vor, beim Liebeswahn und beim Eifersuchtswahn finden sich dagegen keine Verteilungsunterschiede. Noch seltener, mit einer Lebenszeitprävalenz von unter $1 \%$, sind hier nicht gesondert angeführte Themen wie Schwangerschafts- und Verarmungswahn.

Bei all diesen Verteilungsunterschieden sollte nicht übersehen werden, dass sich dahinter Ordnungsstrukturen erkennen lassen, die auf einen kulturunabhängigen Kern des schizophrenen Wahns verweisen (s. Tab. 6). So ist etwa

Tab. 6 Rangordnung der Lebenszeit- und Punktprävalenz von Wahnthemen ( $\mathrm{N}=1.080)$

\begin{tabular}{|c|c|c|c|c|c|c|c|c|c|c|c|c|c|c|}
\hline & \multicolumn{2}{|c|}{ Österreich } & \multicolumn{2}{|c|}{ Polen } & \multicolumn{2}{|c|}{ Litauen } & \multicolumn{2}{|c|}{ Georgien } & \multicolumn{2}{|c|}{ Pakistan } & \multicolumn{2}{|c|}{ Nigeria } & \multicolumn{2}{|c|}{ Ghana } \\
\hline & LP & PP & LP & PP & LP & PP & LP & PP & LP & PP & LP & PP & LP & PP \\
\hline Verfolgung & 1 & 1 & 1 & 1 & 1 & 1 & 1 & 1 & 1 & 1 & 1 & 1 & 1 & 1 \\
\hline Größe & 2 & 3 & 2 & 5 & 2 & 4 & 2 & 4 & 3 & 3 & 2 & 2 & 2 & 2 \\
\hline Religion & 3 & 2 & 3 & 2 & 4 & 2 & 3 & 2 & 4 & 4 & 3 & 3 & 3 & 3 \\
\hline Hypochondrie & 4 & 4 & 5 & 4 & 6 & 7 & 9 & - & 5 & 5 & 6 & 5 & 5 & 5 \\
\hline Schuld & 5 & 6 & 6 & 6 & 5 & 6 & 8 & - & 7 & - & 7 & 9 & 7 & 8 \\
\hline Vergiftung & 6 & 5 & 4 & 3 & 3 & 5 & 4 & 3 & 2 & 2 & 4 & 4 & 4 & 4 \\
\hline Weltuntergang & 7 & 8 & 7 & 7 & 3 & 3 & 10 & 6 & 7 & - & 5 & 6 & 7 & 6 \\
\hline Abstammung & 8 & 7 & 7 & 8 & 7 & 8 & 5 & 5 & - & - & 8 & 7 & 6 & 6 \\
\hline Liebe & 9 & 9 & 8 & - & 9 & - & 6 & 6 & - & - & 9 & 8 & 7 & 7 \\
\hline Eifersucht & 10 & - & 9 & - & 8 & 9 & 7 & 7 & 6 & 5 & 9 & 10 & 8 & - \\
\hline
\end{tabular}

$\mathrm{LP}=$ Lebenszeitprävalenz; PP = Punktprävalenz 
in allen Ländern der Verfolgungs- und Beeinträchtigungswahn das am häufigsten genannte Thema, gefolgt vom Größenwahn.

\subsubsection{Mono- und polythematischer Wahn}

Die Frage nach der Vielgestaltigkeit oder Einförmigkeit des Wahns berührt das alte Paranoiaproblem (Schmidt-Degenhardt 2010). Wahnhafte Störungen oder paranoide Psychosen zeichnen sich durch den Umstand aus, dass der Kranke nahezu vollständig durch eine Thematik okkupiert ist. Der Wahn ist eingekapselt, der überwiegende Teil der psychischen Struktur bleibt intakt (Kretschmer 1918; Stompe u. Schanda 2011). Monothematischer Wahn findet sich allerdings nicht nur bei wahnhaften Störungen, sondern auch bei schizophrenen Erkrankungen. Dieser Umstand führte im 20. Jahrhundert vor allem in der deutschsprachigen Psychiatrie zu zahlreichen Diskussionen über den eigenständigen Status wahnhafter Störungen. Je ausgedehnter ein Wahn ist, daher je mehr Wahnthemen elaboriert sind, desto umfangreicher sind die Strukturverformungen (Janzarik 1988).

Unbekannt war bisher:

- wie häufig monothematische und polythematische Wahngebäude in unterschiedlichen Kulturen vorkommen

- welche Wahnthemen als solitärer Inhalt das Feld okkupieren können

v welche Wahninhalte in polythematischen Wahngebäuden miteinander verbunden sind

Diese Fragestellungen sollen in Folge untersucht werden.

\section{Monothematischer Wahn}

Wie Abbildung 11 zeigt, variiert die Prävalenz des monothematischen Wahns interkulturell deutlich $\left(\mathrm{chi}^{2}=103,5 ; \mathrm{p}=, 000\right)$. Auffällig ist die hohe Rate in Pakistan, wo nahezu von $70 \%$ der Patienten ausschließlich über ein Thema berichtet wurde. Am anderen Ende des Spektrums befindet sich Chana mit lediglich $6 \%$. In den europäischen Staaten schwankte die Prävalenz zwischen 16,6\% (Litauen) und 34,4\% (Österreich).

Mit der Ausnahme von Pakistan finden sich bei schizophrenen Erkrankungen deutlich häufiger polythematische Wahngebäude. Generell sind in Pakistan schizophrene Erkrankungen in vieler Hinsicht einförmiger als in den anderen Ländern: Halluzinatorische Erlebnisse beschränken sich für gewöhnlich auf Phoneme (Bauer et al. 2011), hebephrene Subtypen und Residuen sind häufiger, schizoaffektive und paranoide Subtypen seltener anzutreffen als in den übrigen Ländern (Stompe u. Friedmann 2007).

In der Gesamtgruppe finden sich von den zehn in der ISPS erfassten Wahninhalten nur wenige in monothematischer Form (s. Abb. 12). 


\section{Wahn und Kultur}

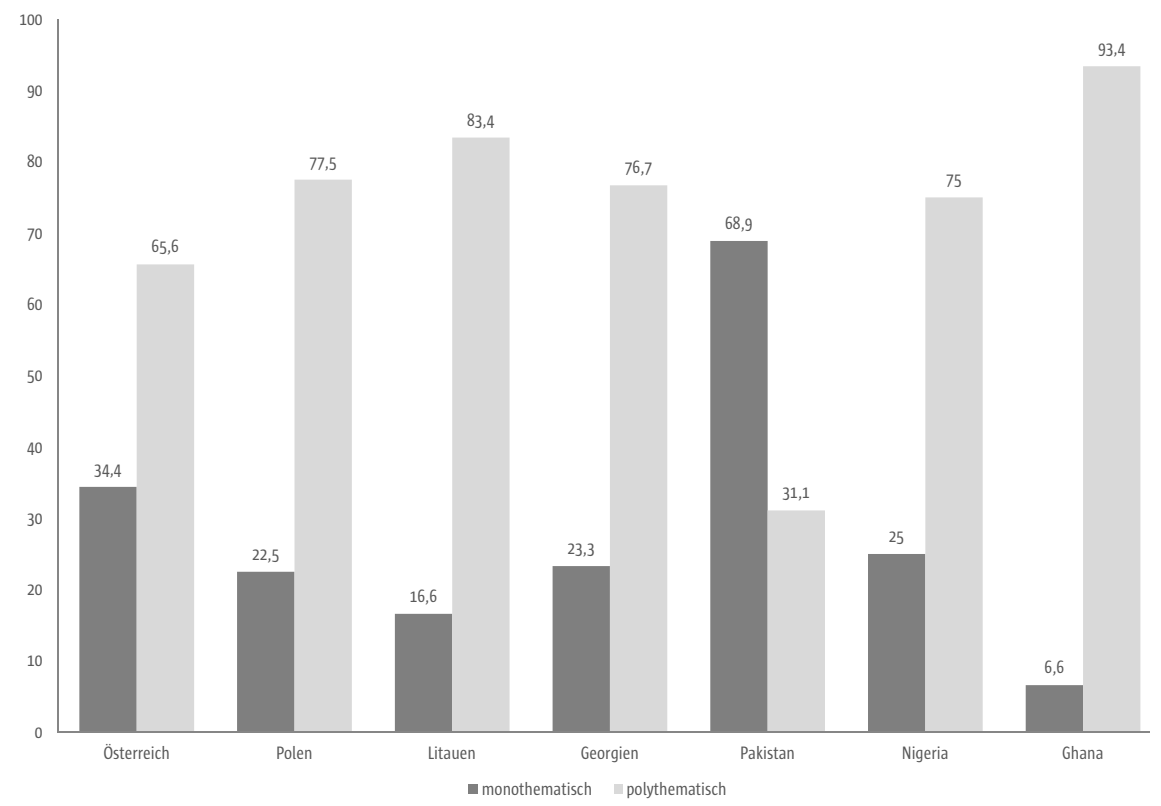

Abb. 11 Häufigkeit von mono- und polythematischem Wahn in \% ( $\mathrm{N}=1.080)$

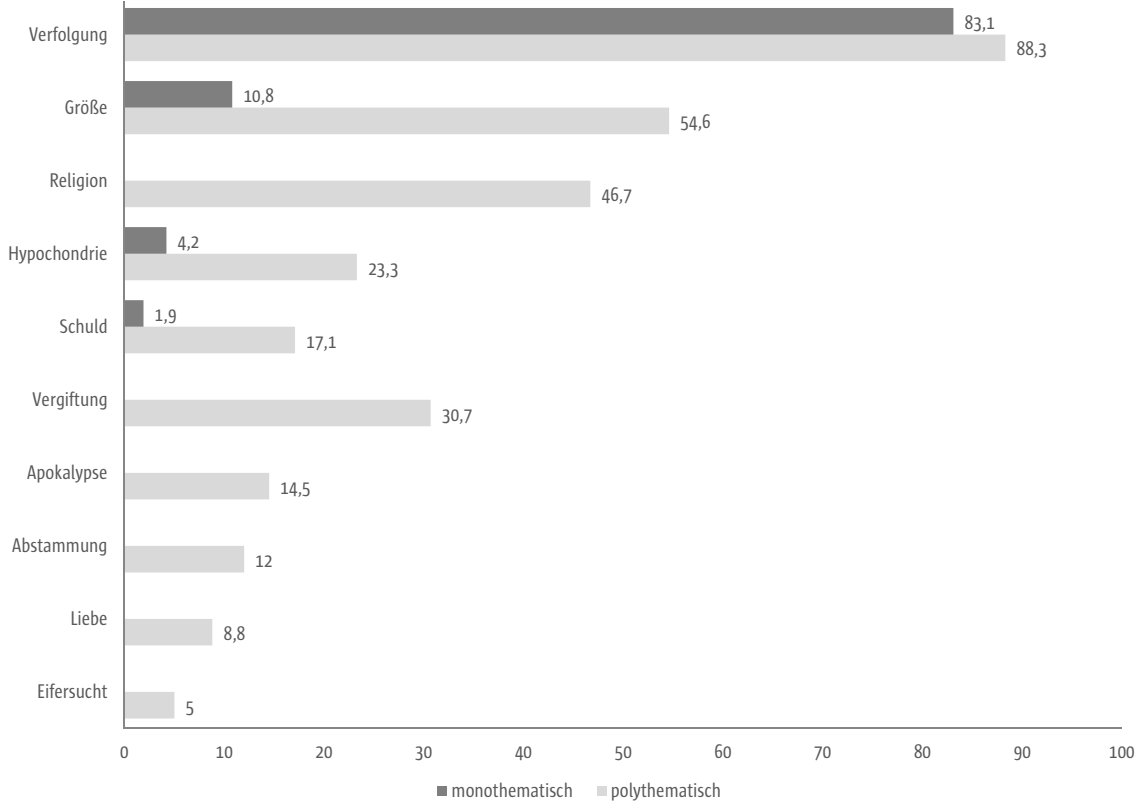

Abb. 12 Prävalenz mono- und polythematischer Wahnthemen in \% in der Gesamtgruppe $(\mathrm{N}=1.080)$ 


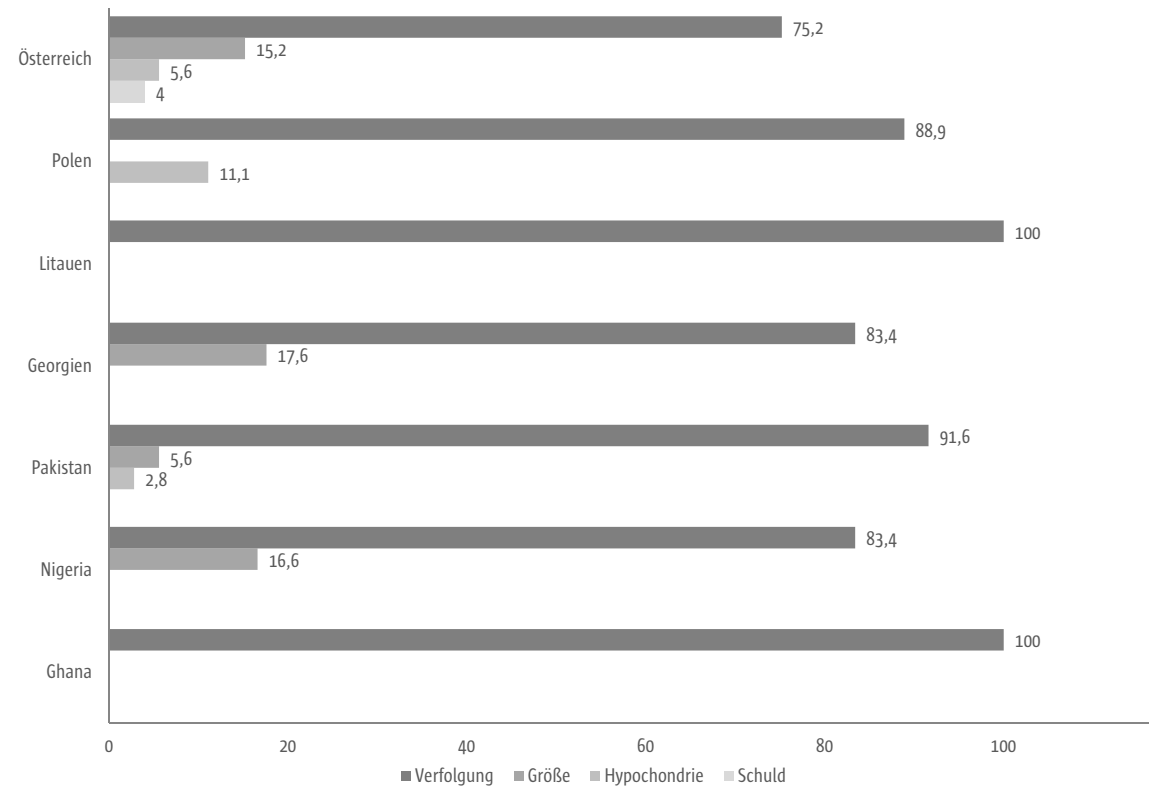

Abb. 13 Monothematische Wahninhalte in \% (N=343)

Auch hier nimmt der Verfolgungswahn die zentrale Position ein. Nur ganz vereinzelt finden sich Patienten, die ausschließlich einen Größenwahn oder einen hypochondrischen Wahn zeigen, monothematischer schizophrener Schuldwahn ist mit fünf österreichischen Patienten eine Rarität. Es scheint also in allen Kulturen die Idee, verfolgt zu werden, häufig als Erklärungsmuster für die psychotische Weltveränderung auszureichen, danach kommt die Wahnarbeit (die kognitive Verarbeitung der Wahnstimmung und der Basissymptome) weitgehend zum Stillstand (s. Abb. 13).

Es folgt nun, nachdem bereits eine gewisse affektive Beruhigung eingetreten ist, in der Regel eine Phase der Konkretisierung des Verfolgungswahns, in welcher der Kranke Klarheit über Identität der Verfolger, Art, Grund und Zweck der Verfolgung gewinnt, ein Prozess, der schrittweise zur Organisierung und Abkapselung des Wahns führt (Stompe et al. 1999a; Stompe u. Schanda 2011).

\section{Polythematischer Wahn}

Polythematische Wahngebäude finden sich am häufigsten in Chana (93,4\%) sowie in den neuen EU-Staaten Polen $(77,5 \%)$ und Litauen $(83,4 \%)$. Nur wenig darunter liegt die Rate in Nigeria (75\%) und in Georgien $(76,7 \%)$, gefolgt von Österreich $(65,6 \%)$ (s. Abb. 11). In den meisten Ländern nennt die überwiegende Zahl der Patienten mit polythematischem Wahn im Durchschnitt 3,o \pm 1,2 Wahnthemen (s. Abb. 14). 


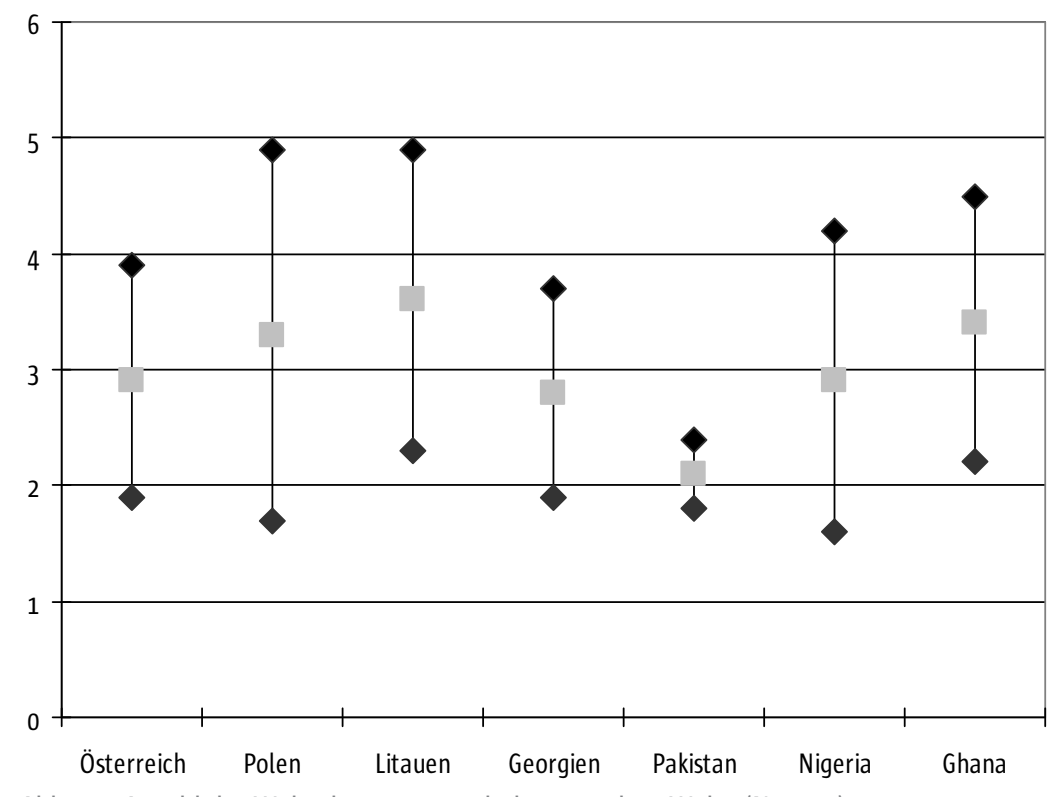

Abb. 14 Anzahl der Wahnthemen im polythematischen Wahn ( $N=737)$

Ausnahme ist Pakistan mit im Durchschnitt 2,1 $\pm 0,3$ Themen. Wie zuvor gezeigt wurde, ist Pakistan das einzige der untersuchten Länder, in dem wir häufiger monothematische als polythematische Wahngebäude antrafen. Dieser Trend scheint sich somit in der geringeren Anzahl von Wahnthemen im polythematischen Kontext fortzusetzen.

Die Prävalenzen sämtlicher Wahnthemen sind im polythematische naturgemäß höher als im monothematischen Wahn (s. Abb. 12). Betroffen davon ist vor allem der Crößenwahn, der monothematisch lediglich von 10,8\%, polythematisch hingegen von 54,6\% der Befragten angegeben wurde. Religiöse Wahninhalte, Vergiftungs- und apokalyptische Ideen sowie Abstammungs-, Liebes-, und Eifersuchtswahn fanden sich ausschließlich in polythematischer Fassung. Insgesamt ist die Vielfalt der Wahnthemen schizophrener Erkrankungen mit der Ausnahme von Pakistan auf polythematische Wahngebäude zurückzuführen. Wie besprochen nimmt der monothematische Verfolgungs- und Beeinträchtigungswahn kulturunabhängig eine herausragende Stellung ein (s. Abb. 13). Die wahnhafte Gewissheit, verfolgt zu werden, ist allerdings auch im polythematischen Kontext mit großem Abstand die am häufigsten genannte Thematik. Die Differenz der Prävalenzen von monothematischem und polythematischem Verfolgungswahn ist zudem deutlich geringer als bei allen anderen Wahnthemen.

Mit Ausnahme des Liebeswahns finden sich bei allen Wahnthemen interkulturell statistisch signifikante Verteilungsunterschiede, die vor allem auf die besondere thematische Zusammensetzung des Wahns in Pakistan zurückzuführen sind (s. Tab. 7). 
Tab. 7 Polythematische Wahninhalte in $\%(\mathrm{~N}=737)$

\begin{tabular}{l|rrrrrrr} 
& $\begin{array}{c}\text { Österreich } \\
(\mathrm{n}=238)\end{array}$ & $\begin{array}{c}\text { Polen } \\
(\mathrm{n}=62)\end{array}$ & $\begin{array}{r}\text { Litauen } \\
(\mathrm{n}=61)\end{array}$ & $\begin{array}{c}\text { Georgien } \\
(\mathrm{n}=57)\end{array}$ & $\begin{array}{c}\text { Pakistan } \\
(\mathrm{n}=32)\end{array}$ & $\begin{array}{c}\text { Nigeria } \\
(\mathrm{n}=233)\end{array}$ & $\begin{array}{c}\text { Ghana } \\
(\mathrm{n}=71)\end{array}$ \\
\hline Verfolgung & $86,1 \%$ & $90,3 \%$ & $98,4 \%$ & $73,7 \%$ & $93,8 \%$ & $80,6 \%$ & $98,6 \%$ \\
\hline Größe & $59,2 \%$ & $53,2 \%$ & $50,8 \%$ & $43,9 \%$ & $21,9 \%$ & $49,4 \%$ & $69,0 \%$ \\
\hline Religion & $52,5 \%$ & $43,5 \%$ & $36,1 \%$ & $36,8 \%$ & $25,0 \%$ & $50,2 \%$ & $36,8 \%$ \\
\hline Hypochondrie & $23,5 \%$ & $33,9 \%$ & $27,9 \%$ & $17,5 \%$ & $9,4 \%$ & $5,6 \%$ & $29,6 \%$ \\
\hline Schuld & $18,9 \%$ & $24,2 \%$ & $29,5 \%$ & $7,0 \%$ & $3,1 \%$ & $13,9 \%$ & $9,9 \%$ \\
\hline Vergiftung & $16,4 \%$ & $40,3 \%$ & $39,3 \%$ & $3,5 \%$ & $3,1 \%$ & $19,4 \%$ & $9,9 \%$ \\
\hline Apokalypse & $12,6 \%$ & $16,1 \%$ & $18,0 \%$ & $29,8 \%$ & - & $11,1 \%$ & $11,3 \%$ \\
\hline Abstammung & $7,1 \%$ & $16,1 \%$ & $18,0 \%$ & $29,8 \%$ & - & $11,1 \%$ & $11,3 \%$ \\
\hline Liebe & $7,6 \%$ & $14,5 \%$ & $4,9 \%$ & $15,8 \%$ & - & $8,3 \%$ & $9,9 \%$ \\
\hline Eifersucht & $3,4 \%$ & $1,6 \%$ & $13,1 \%$ & $8,9 \%$ & $3,1 \%$ & $8,3 \%$ & $2,8 \%$ \\
\hline
\end{tabular}

Wie schon der Überblick über die Punkt- und Lebenszeitprävalenz gezeigt hat, berichteten pakistanische Patienten deutlich seltener über religiöse, Größen-, schuld- und hypochondrische Ideen. Monothematisch und polythematisch steht die negative Veränderung der Welt (Verfolgungs- und Vergiftungswahn) ganz im Zentrum des Wahns (s. Tab. 5). Wie in einer früheren Arbeit gezeigt wurde (Stompe et al. 1999a), stehen Verwandte und Partner als Verfolger im Zentrum der wahnhaften Ängste. Das stützende, aber auch fordernde und oft überfordernde hierarchisch organisierte Familiensystem führt im Vorfeld der Psychose oftmals zu Konflikten, da vor allem die ältesten Söhne die in sie gesetzten normativen Erwartungen nicht erfüllen können (Stompe et al. 1999b), eine Konstellation, die zu einer Fixierung der Wahnthematik auf Verfolgung, Beeinträchtigung und Vergiftung führen kann.

Zur Beantwortung der Frage, ob die polythematisch strukturierten Wahninhalte überall gleichförmig miteinander verbunden sind oder ob sich hier kulturspezifische Unterschiede ausmachen lassen, wurden hierarchische Clusteranalysen gerechnet, deren Ergebnisse zur besseren Illustration als Dendrogramme dargestellt werden. Die sieben Einzelabbildungen der Abbildung 15 veranschaulichen diese Zusammenhänge.

Auch hier wird einmal mehr die Bedeutung des Verfolgungswahns bei schizophrenen Erkrankungen evident. In den osteuropäischen Ländern bilden alle übrigen Themen einen Cluster, der mit dem Verfolgungswahn verbunden ist. In Österreich, Pakistan und den beiden westafrikanischen Ländern findet sich eine 2-Cluster Lösung. Wiederum nimmt der Verfolgungswahn die zentrale Position im Cluster 1 ein. Größenwahn und religiöser Wahn treten regelhaft gemeinsam auf. Im Gegensatz zu manischen Größenideen hat der schizophrene Größenwahn offenbar sehr häufig eine religiöse Färbung. Mit der Ausnahme 

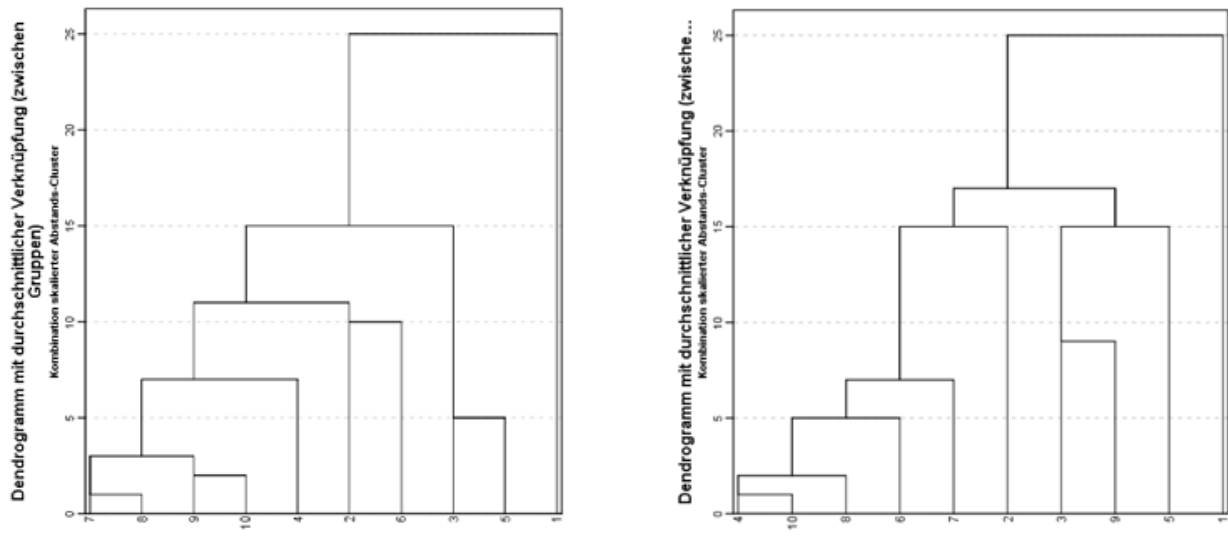

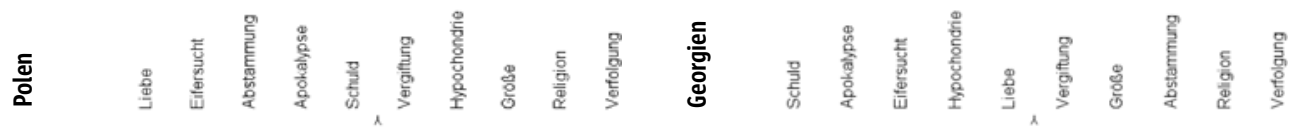
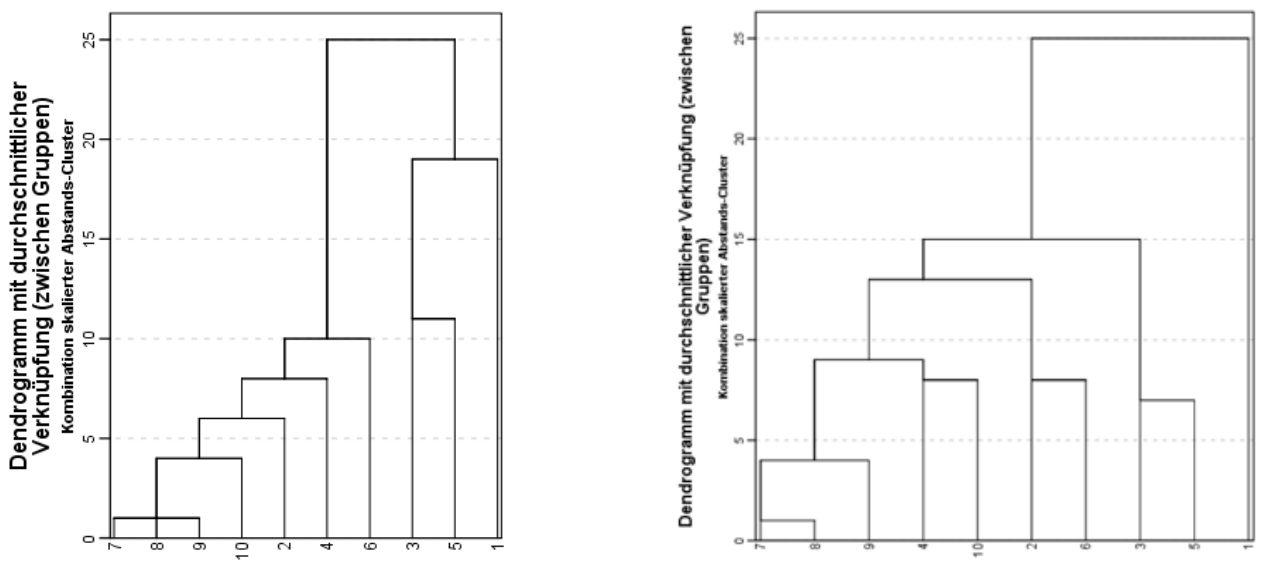

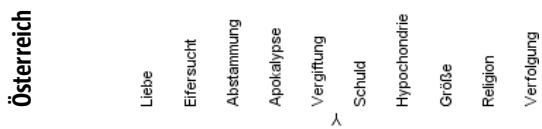

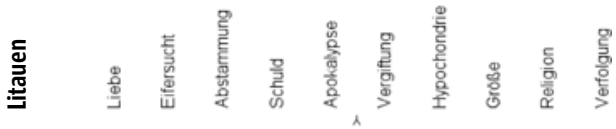



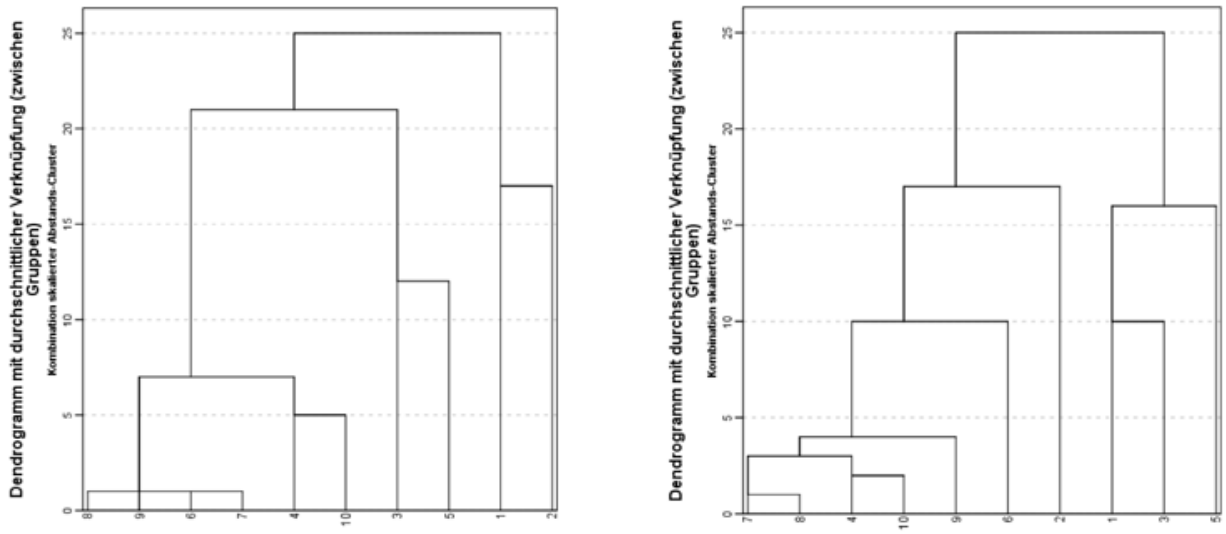

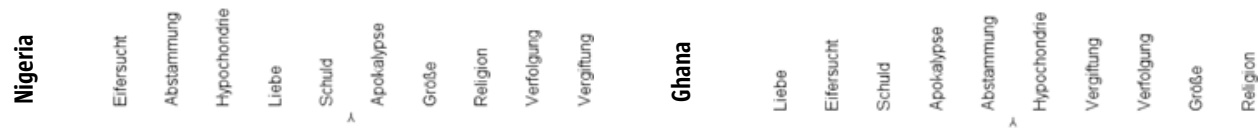

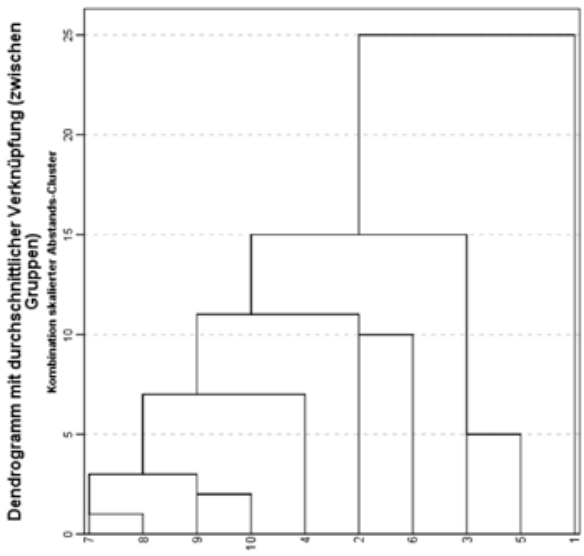

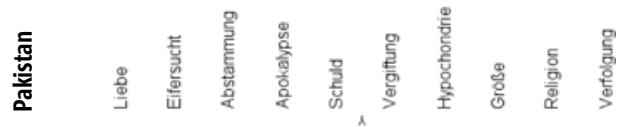

Abb. 15 Polythematische Wahncluster $(N=737)$ 
von Ghana sind Verfolgungsideen dagegen überwiegend profan. So findet sich sehr häufig eine Konstellation, in der der Kranke eine religiöse Wahnidentität (z.B. Jesus) hat, gleichzeitig aber meint, von seiner (profanen) Umwelt verfolgt zu werden. In Österreich, Polen und Litauen bilden wahnhafte Eifersuchts- und Liebesthemen, die beide auf reale (Eifersuchtswahn) oder imaginierte (Liebeswahn) Intimbeziehungen verweisen, einen Mikrocluster. Die anderen Wahnthemen sind interkulturell variabel miteinander verbunden. Manchmal treten apokalyptischer und Schuldwahn gemeinsam auf(Litauen, Georgien, Nigeria und Ghana). Leicht nachvollziehbar ist die Assoziation von Vergiftungswahn mit Verfolgungswahn (Pakistan, Nigeria) bzw. mit hypochondrischem Wahn (Polen, Litauen).

\subsection{Struktur des Wahns}

Unter Wahnstruktur werden Organisation (Systematisierung), Logik, und Aufbauelemente des Wahns verstanden (Berner 1982; Stompe u. Schanda 2011). Die Begriffe organisiert (systematisiert), bzw. unorganisiert (unsystematisiert) kennzeichnen, inwieweit der Wahn zu einem geschlossenen Gebilde zusammengefasst ist oder nicht (Berner 1982). Unter Organisation versteht man das Bemühen des Betroffenen, durch Wahnarbeit einen zunächst unorganisierten Wahn zu ordnen. Gelingt dies, so wird das daraus resultierende straff organisierte Wahngebäude als systematisierter Wahn bezeichnet. Die Analyse der Wahnlogik bezeichnet die logische Struktur der Ideenverknüpfungen des Wahns. Aufbauelemente sind Phänomene aus welchen sich der Wahn konstituiert. Diese können im Verlauf aber auch seiner Ausgestaltung dienen. Aufbauelemente können sowohl normopsychische Akte (normale Wahrnehmungen, unverfälschte Erinnerungen) als auch unstrukturierte psychotische Erlebnisse (Wahnstimmung und Anmutungen) oder figurale psychotische Phänomene (Trugerinnerungen und Erinnerungsfälschungen, Schneider'sche Erstrangsymptome, verschiedene Formen psychotischer und nicht-psychotischer Wahrnehmungsveränderungen und Konfabulationen) sein. Im Folgenden werden wir uns auf die Darstellung der Organisation und der Wahnlogik bei monothematischen und polythematischen Wahn beschränken.

\subsubsection{Organisation (Systematisierung) des Wahns}

Während ein monothematischer Wahn zumeist organisiert (systematisiert) ist, liegt polythematischer Wahn sowohl in organisierter als auch in unorganisierter Form vor (s. Abb. 16).

In den europäischen Ländern ist der monothematische Wahn in 84-94\% der Fälle organisiert, in Pakistan sogar in 98\%. Etwas niedriger ist diese Rate in den westafrikanischen Staaten. Hier ist der monothematische Wahn bei $28 \%$ der nigerianischen und bei immerhin $37 \%$ der ghanaischen Patienten unorganisiert. 


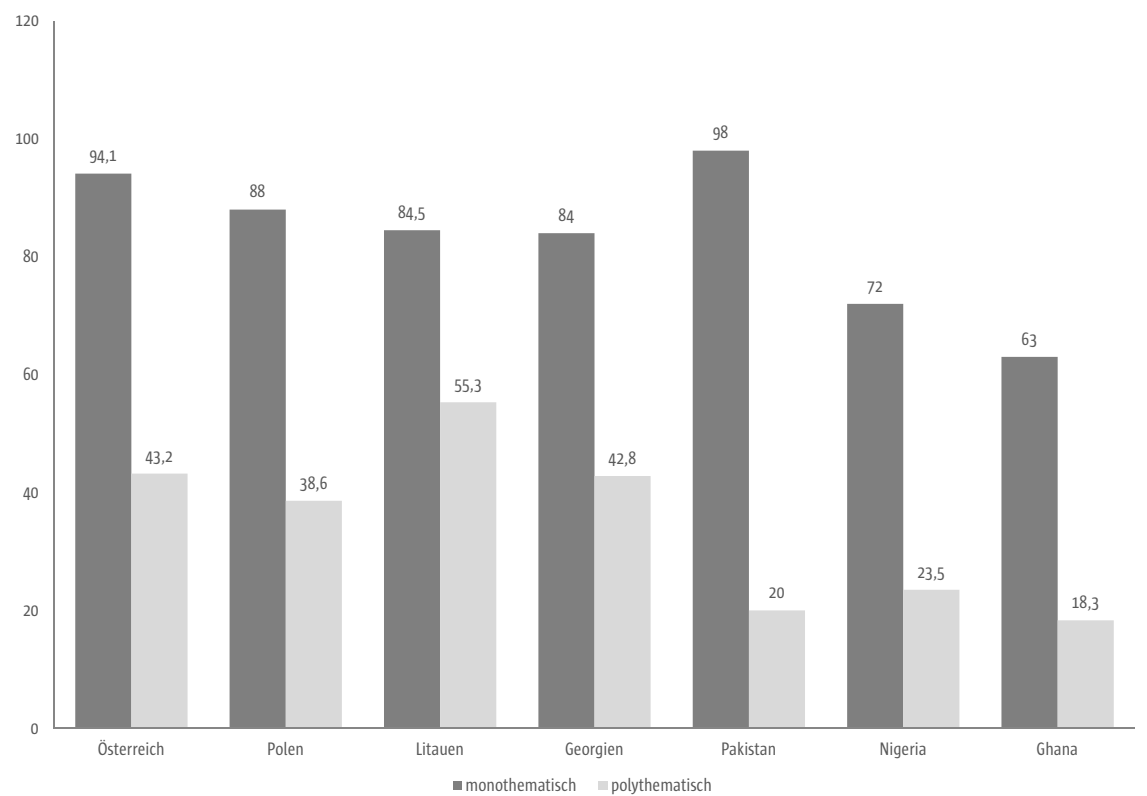

Abb. 16 Häufigkeit von organisiertem monothematischem und polythematischem Wahn in \% $(\mathrm{N}=1.080)$

Umgekehrt sind in nahezu allen Ländern polythematische Wahnbildungen vorwiegend unorganisiert. In den außereuropäischen Staaten ist der Wahn deutlich öfter unorganisiert als in Europa. Mehrere gleichzeitig vorhandene Wahnthemen scheinen die kognitiven Integrationsleistungen manchmal zu überfordern. Wenn hingegen nur ein Wahnthema bearbeitet werden muss, entsteht in der überwiegenden Zahl ein organisierter Wahn. Der westliche Zwang zur geschlossenen Form könnte die höhere Prävalenz von organisiertem polythematischem Wahn in den europäischen Staaten erklären.

\subsubsection{Wahnlogik}

Eine ähnliche Konstellation findet sich beim logischen Aufbau des Wahns. Auch hier ist der monothematische Wahn, vor allem in den europäischen Staaten und in Pakistan häufig in sich logisch strukturiert. In Nigeria und in Ghana sind aber immerhin in 36\% bzw. 50\% der monothematischen Wahngebäude paralogisch (s. Abb. 17).

Die Anzahl der logisch strukturierten polythematischen Wahnformationen ist in allen Kulturen deutlich geringer. Besonders in den nicht-europäischen Staaten findet sich vorwiegend einen paralogisch aufgebauter polythematischer Wahn (Pakistan: 88\%, Nigeria: 87,3\%, Chana: 86,3\%), während in Europa hier das Verhältnis von logisch zu paralogisch ausgeglichener ist. 


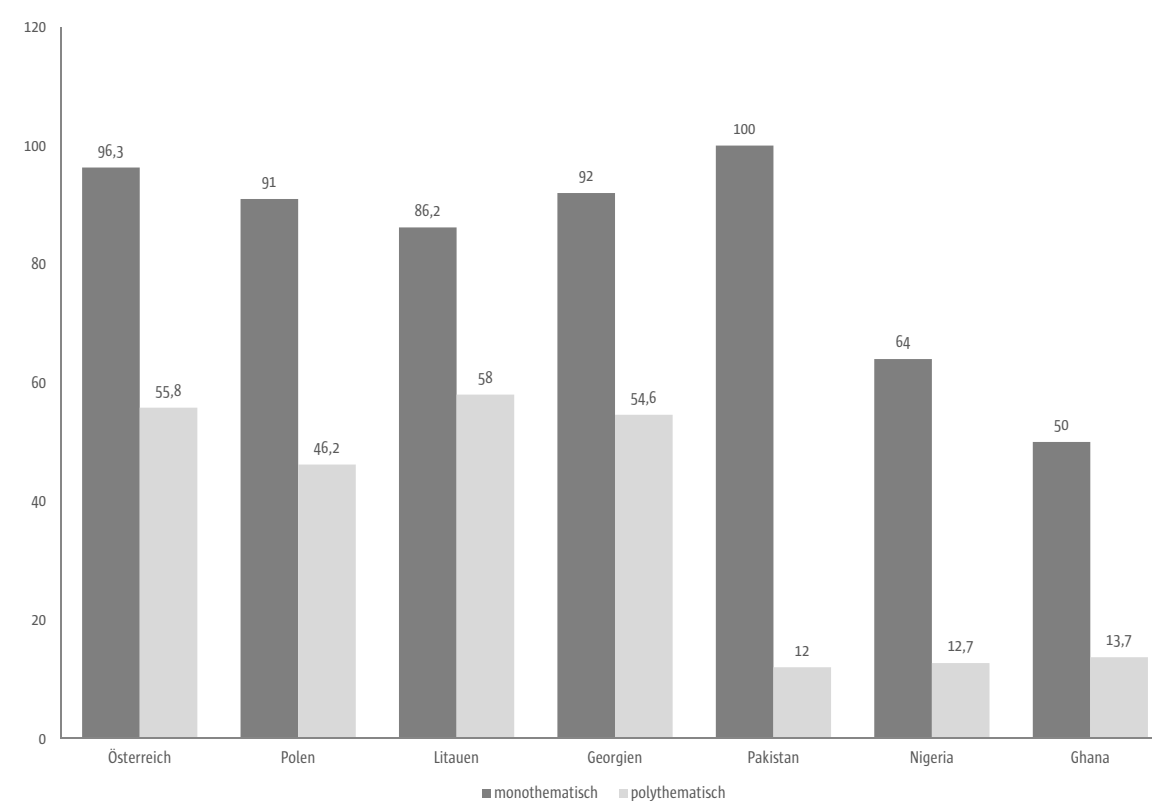

Abb. 17 Häufigkeit von logischem monothematischem und polythematischem Wahn in \% $(\mathrm{N}=1.080)$

Vor allem in Pakistan wird beim Vergleich von monothematischem (logisch: 100\%) und polythematischem Wahn (logisch: 12\%) deutlich, dass es den Kranken häufig nicht gelingt, mehreren Wahnthemen zugleich logisch durchzuarbeiten. Im westafrikanischen Kulturraum scheinen logische Widersprüche weniger problematisch zu sein. Der Wahn zeigt in Nigeria und Ghana deutlich häufiger eine paralogisch-unorganisierte Struktur als in den anderen Ländern.

\section{Zusammenfassung}

Um Wahnthemen kulturvergleichend untersuchen zu können, ist eine kategoriale Wahndefinition erforderlich. Diese Definition darf sich nicht auf inhaltliche Kriterien wie die nach Jaspers oder DSM beziehen. Das Vorliegen eines Wahns kann dann als valide und reliabel angenommen werden, wenn Untersucher aus verschiedenen Kulturen mit zwei Definitionen Erlebnisse als Wahn klassifizieren.

Wahnthemen lassen sich in ein bipolar angeordnetes dreidimensionales Koordinatensystem einordnen, das auf eine semantische Ordnung der Wahninhalte verweist. In allen untersuchten Kulturen sind Verfolgungs- und Größenwahn die häufigsten schizophrenen Wahnthemen. Im Längsschnitt findet sich eine bunte Fülle an Wahnthemen, im Querschnitt ist die Erkrankung deutlich einförmiger. Besonders auffällig wird dies bei monothematischen Wahngebäuden, der nahezu ausschließlich aus Verfolgungsideen besteht. Polythematisch ist der inhaltliche Aufbau auch im Querschnitt wesentlich vielfältiger. Die Clusteranalyse weist hier auf basale kulturunabhängige Ordnungsprinzipien der Wahnthemen hin. 
Daneben gibt es allerdings auch deutliche interkulturelle Unterschiede: Schuldwahn etwa findet sich vorwiegend in Ländern mit einer überwiegend christlichen Bevölkerung. In den westafrikanischen Staaten ist der Wahn häufiger religiös gefärbt, unorganisiert und paralogisch.

Wie wir zu zeigen versuchten, kann die kulturvergleichende Psychiatrie zu vertieften Einsichten in das Wesen und den grundlegenden Aufbau des Wahns verhelfen. Auf Zudem finden sich auch zahlreiche Hinweise auf den pathoplastischen Effekt von Kultur und Gesellschaft.

\section{Literatur}

Bauer S, Schanda H, Karakula H, Rudaleviciene P, Okribelashvili N, Chaudhry HR, Idemudia I, Gscheider S, Ritter K, Stompe T (2011) Culture and the prevalence of hallucinations in schizophrenia. Comprehensive Psychiatry in Druck

Berner P (1982) Psychiatrische Systematik. Huber Bern Stuttgart Wien

Blankenburg W (Hrsg.) (1991) Wahn und Perspektivität. Enke Stuttgart

Bleuler E (1911) Dementia praecox oder die Gruppe der Schizophrenien. Deuticke Leipzig Wien

Brandom R (2004) Die expressive Vernunft. Suhrkamp Frankfurt am Main

Conrad K (1958) Die beginnende Schizophrenie. Thieme Stuttgart

Gruhle H (1951) Über den Wahn. Nervenarzt 22, 125-126

Huber G, Gross G (1977) Wahn. Enke Stuttgart

Janzarik W (1988) Strukturdynamische Grundlagen der Psychiatrie. Enke Stuttgart

Jaspers K (1913) Allgemeine Psychopathologie. Springer Berlin Heidelberg New York

Jossmann P (1921) Das Problem der Überwertigkeit. Z Ges Neurol Psychiatr 64, 1-82

Kala AK, Wig NN (1982) Delusion across cultures. Int / Soc Psychiatry 28,185-193

Kim K, Hwu H, Zhang LD, Lu MK, Park KK, Hwang T], Kim D, Park YC (2001) Schizophrenic delusions in Seoul, Shanghai and Taipei: a transcultural study. I Korean Med Sci 16, 88-94

Kraepelin E (1899) Ein kurzes Lehrbuch für Studirende und Aerzte. 6., vollständig umgearbeitete Auflage. Barth Leipzig

Kranz H (1955) Das Thema des Wahns im Wandel der Zeit. Fortschr Neurol Psychiatr 23, 829-827

Kretschmer E (1950) Der sensitive Beziehungswahn. 3. Auflage. Springer Berlin

Lenz H (1964) Vergleichende Psychiatrie. Eine Studie über die Beziehung von Kultur, Soziologie und Psychopathologie. Maudrich Wien

Mastowski I (1986) Pathoplastic influences on symptoms of schizophrenia. A comparative study in Libya and Malta. Acta Psychiatr Scand 73, 618-623

Mittelstraß | (Hrsg.) (1995) Enzyklopädie und Wissenschaftstheorie. Verlag I.B. Metzler Stuttgart Weimar

Ndetei DM, Vadher A (1984) Frequency and clinical significance of delusions across cultures. Acta Psychiatr Scand $70,73-76$

Saß H, Wittchen H-U, Zaudig M (1996) Diagnostisches und Statistisches Manual psychischer Störungen DSM-IV. Göttingen Bern Toronto Seattle

Schmidt-Degenhard M (2010) Die Paranoiafrage - Problemgeschichtliche und psychopathologische Überlegungen. In: Lammel M, Sutarski S, Lau S, Bauer M (Hrsg.) Wahn und Schizophrenie. Psychopathologie und forensische Relevanz. 33-46. Medizinischer Wissenschaftsverlag Berlin

Spitzer M (1989) Ein Beitrag zum Wahn. Nervenarzt 60, 95-101

Stompe T (2008) Geschlecht, Alter und Wahnthematik in der Schizophrenie. / Neurol, Neurochir Psychiatr 2, 35-37

Stompe T (2010) Problemstellungen und Ergebnisse der Transkulturellen Wahnforschung. Psychopraxis 13, 22-27

Stompe T, Bauer S, Karakula H, Rudaleviciene P, Okribelashvili N, Chaudhry HR, Idemudia EE, Gscheider S (2007) Paranoid-hallucinatory syndromes in schizophrenia - results of the International Study on Psychotic Symptoms. WCPRR 4, 63-68 
Stompe T, Bauer S, Ortwein-Swoboda G, Schanda H, Karakula H, Rudaleviciene P, Chaudhry HR, Idemudia ES, Gschaider S (2006a) Delusions of guilt: The attitude of Christian and Islamic confessions towards Good and Evil and the responsibility of men. I Muslim Mental Health 1, 43-56

Stompe T, Friedman A, Ortwein G, Strobl R, Chaudhry HR, Najam N, Chaudhry MR (1999a) Comparison of delusions among schizophrenics in Austria and in Pakistan. Psychopathology 32, 225-234

Stompe T, Friedmann A (2007) Culture and Schizophrenia. In: Bhugra D (Hrsg.) Textbook for Cultural Psychiatry. 314-322. Cambridge University Press Cambridge

Stompe T, Karakula H, Rudaleviciene P, Okribelashvili N, Chaudhry HR, Idemudia EE, Gscheider S (2006b) The pathoplastic effect of culture on psychotic symptoms in schizophrenia. WCPRR 1, 157-163

Stompe T, Ortwein-Swoboda G, Friedmann A, Chaudhry HR (199gb) Sibling orders of schizophrenic patients in Austria and Pakistan. Psychopathology 32, 281-291

Stompe T, Ortwein-Swoboda G, Ritter K, Schanda H (2003) Old wine in new bottles? Stability and plasticity of the contents of schizophrenic delusions. Psychopathology 36, 6-12

Stompe T, Schanda H (2011) Die klinisch-deskriptive Psychopathologie des Wahns. In: Unterthurner G, Kadi U (Hrsg.) Wahn. Philosophische, psychoanalytische und kulturwissenschaftliche Perspektiven. Turia + Kant Wien in Druck

Suhail K, Cochrane R (2002) Effect of culture and environment on the phenomenology of delusions and hallucinations. Int | Soc Psychiatry 48, 126-138

Tateyama M, Asai M, Hashimoto M, Bartels M, Kasper S (1998) Transcultural study of schizophrenic delusions. Tokyo versus Vienna and Tübingen (Germany). Psychopathology 31, 59-68

Tateyama M, Asai M, Kamisada M, Hashimoto M, Bartels M, Heimann H (1993) Comparison of schizophrenic delusions between Japan and Germany. Psychopathology 26, 151-158 